\title{
Stimulus presentation frequency in brightness discrimination and generalization: A test of adaptation-level and signal-detection interpretations
}

\author{
DAVID R. THOMAS and BRADLEY T. WINDELL \\ University of Colorado, Boulder, Colorado \\ JON L. WILLIAMS \\ Kenyon College, Gambier, Ohio \\ and \\ K. GEOFFREY WHITE \\ Victoria University of Wellington, Wellington, New Zealand
}

\begin{abstract}
In Experiment 1, brightness discriminations were established under conditions in which the stimulus to which "same" responses were required (S+) was presented more frequently than the stimulus to which "different" responses were made (S-), S+ and S- were presented equally often, or S- was more frequent than S+. Subsequent generalization gradients along the brightness dimension were shifted away from the stimulus value more frequently presented during training. In Experiment 2, brightness discriminations were established under conditions of equally frequent presentations of $\mathrm{S}+$ and $\mathrm{S}-$ stimuli in training, but in subsequent generalization testing $\mathrm{S}+$ and $\mathrm{S}$ - values were equally represented or overrepresented relative to presentations of other test stimuli. Generalization gradients shifted towards the overrepresented stimulus value, relative to gradients obtained for equal presentation-frequency conditions. The shift in gradients away from the more frequently presented stimulus during training in Experiment 1 was consistent with accounts of stimulus generalization based on both adaptation-level theory and signal-detection theory. The shift in gradients towards the more frequently presented stimulus during testing in Experiment 2, however, was consistent with adaptation-level theory, whereas the signaldetection account had predicted a shift in the opposite direction. These results attest to the importance of relative stimulus presentation frequency as a determinant of postdiscrimination stimulus generalization performance. Because feedback was given only during training, the opposite effects of presentation probability in training and testing may reflect an interaction between presentation probability and feedback.
\end{abstract}

In the study of stimulus generalization with humans as subjects, verbal instructions to respond to a particular (training) stimulus value (and to no other) are often substituted for the operant or Pavlovian training given to infrahuman organisms (Brown, Bilodeau, \& Baron, 1951; Kalish, 1958). The gradients obtained, however, look much like those obtained in conditioning studies; that is, typically maximal responding occurs to the value of the training stimulus, with response probability systematically decreasing as a function of the dimensional distance between a given test stimulus and the training value.

When subjects are instructed or trained to respond to one stimulus value $(\mathrm{S}+)$ but not to another $(\mathrm{S}-$ ) on the

D. R. Thomas's mailing address is: Department of Psychology, Muenzinger Psychology Building, Campus Box 345, University of Colorado, Boulder, CO 80309. same sensory continuum, a typical result, in humans as in other animals, is an asymmetrical postdiscrimination generalization gradient with depressed responding in the vicinity of $\mathbf{S}$ - and enhanced responding to stimuli on the side of $\mathbf{S}+$ opposite the $S-$. This enhanced responding is called an "area shift" except when the enhancement is sufficient to displace the point of maximal responding, in which case the effect is labeled a "peak shift." Area shift and peak shift are thought to reflect the same underlying process, with an area shift seen in the group average of gradients, only some of which included a displacement in peak responding. The extensive literature on this shift phenomenon in animals has been reviewed by Purtle (1973), and studies of peak shift in humans have been reviewed by Thomas (1974). In the first study with human subjects, Doll and Thomas (1967) used a monochromatic light of $530 \mathrm{~nm}$ as S+ and one of $550 \mathrm{~nm}$ as $S-$, with trial-by-trial feedback on correctness. Subse- 
quent generalization testing without feedback revealed a gradient that peaked at $520 \mathrm{~nm}$. Peak shifts have subsequently been demonstrated with such diverse stimulus dimensions as visual intensity (Thomas, Svinicki, \& Vogt, 1973) and auditory frequency (Baron, 1973).

Postdiscrimination peak shifts in the animal literature are typically interpreted in terms of Spence's (1937) gradient interaction theory. The algebraic summation of an excitatory gradient around $\mathbf{S}+$ and an inhibitory gradient around S-leads to a net excitatory gradient with a displaced peak. Galizio and Baron (1979) have argued that Spence's learning interpretation may also apply to peak shift in adult humans. The use of instructions in human studies, however, suggests that a perceptual interpretation of peak shift may be more appropriate. That is, given an instruction not to respond (or to respond in a different fashion) to stimuli that are perceived as different, generalized responses or errors must reflect a failure to perceive a difference, barring the operation of a response bias.

The perceptual account of peak shift offered by Thomas et al. (1973) employed the concepts of adaptation-level theory (Helson, 1964). According to this theory, subjects perceive and remember stimuli not as absolute values but in relation to some internal representation of the "average," or adaptation-level (AL) of previously experienced stimuli on that dimension. Thomas et al. suggested that, during discrimination training, the AL was established between the $S+$ and $S-$ values and subjects used a response rule specifying responses to a value (i.e., $\mathrm{S}+$ ) displaced from the AL. In generalization testing, the previous $\mathrm{S}+$ is typically located at the center of the series of test values and the AL should drift toward this value as the test progresses. Continued use of the response rule during testing would result in responding to a stimulus value displaced from $\mathrm{AL}$, thus yielding a peak shift in the generalization gradient.

Although changes in AL between training and testing values can contribute to peak shift (Newlin, Rodgers, \& Thomas, 1979; Thomas et al., 1973), recent studies show that peak shifts can be obtained when AL remains constant. For example, Galizio and Baron (1973) trained subjects to respond to two $S+s$ (tones varying in frequency) with S- midway between them. Although the ALs of training and test series were the same, a generalization test centered on $\mathrm{S}-$ produced a gradient with a minimum at $S-$ and increased responding in both directions as stimuli became more different from $S-$. Furthermore, even if an AL shift had occurred, it could not account for the bidirectional peak shift.

A bidirectional peak shift was also found in a threestimulus visual intensity discrimination study by White and Thomas (1979). Furthermore, Newlin et al. (1979) obtained evidence for two independent factors that contribute to peak shift, one of which was a change in $\mathrm{AL}$ from training to test values. The question that remains is how best to characterize the second (non-AL) factor.
Newlin et al. (1979) have offered an approach based on the theory of signal detectability (TSD) (Green \& Swets, 1974). According to this theory, any given physical stimulus produces a subjective experience called a discriminal process. Because the discriminal process is variable, repeated presentations of the same physical stimulus generate a discriminal distribution. The subject must then establish a response or decision criterion (or criteria) defining a range of the discriminal distribution that the subject will attribute to a particular stimulus. Boneau and Cole (1967) and Blough (1969) have shown that peaked generalization gradients would be found if instructions (for human subjects) or single stimulus training (for animal subjects) caused subjects to create two symmetrical response criteria around the mean discriminal process produced by the training stimulus. Blough also noted that peak shift or asymmetrical gradients could be accounted for by assuming that the two criteria were independent. Specifically, a peak shift, or more generally an asymmetrical gradient with enhanced responding on the side of S+ opposite the S-, would result if discrimination training caused the criterion on the $S-$ side of $S+$ to be moved toward the mean of the discriminal distribution produced by $\mathrm{S}+$ and had little effect on the opposite criterion. This would mean that stimuli just to the opposite side of $\mathrm{S}+$ from $S$ - would be more likely to produce a discriminal process that would be interpreted as $S+$ than those on the $S-$ side or (in the case of peak shift) the $S+$ value itself.

The TSD approach to the study of stimulus generalization suggests the importance of some procedural variables that might not otherwise be considered. For example, according to TSD, the major determinant of the location of response criteria is the relative frequency of presentation of the training stimuli (Dusoir, 1975, 1983; Green \& Swets, 1974; Schulman \& Greenberg, 1970). When one stimulus is presented more often than the other, the criterion is shifted farther from the mean of the discriminal distribution produced by this stimulus, and thus closer to the mean of the distribution caused by the other stimulus. Therefore, the subject will be more likely to attribute a given sensation to the presentation of the more probable stimulus. This should result in a shift of the postdiscrimination generalization gradient. This prediction was examined recently in a study by Thomas and Vogt (1983). The training stimuli were different intensities of white light, and, in different groups, either the S+ or the $\mathrm{S}-$ was presented on $75 \%$ of the training trials. Overrepresentation of $S+$ resulted in a shift of the gradient towards stimuli in the region of $\mathrm{S}-$, and overrepresentation of $S-$ shifted the gradient towards $S+$. These effects were reflected in an increase or decrease, respectively, in responding to stimuli intermediate between $\mathbf{S}+$ and $\mathbf{S}-$, as well as in displacement of the gradient peak.

Experiment 1 of the present study is an extension of the Thomas and Vogt (1983) experiment. The lack of an equal-representation control group in their study made it impossible to determine whether the overrepresentation 
of both $\mathrm{S}+$ and of $\mathrm{S}-$ were effective in changing the locus of the decision criterion. Another change was that the training values used in Experiment 1 were more widely spaced in the hope that this would increase the size of the observed effects.

\section{EXPERIMENT 1}

\section{Method}

Subjects. The subjects were 120 students enrolled in introductory psychology classes at the University of Colorado. Of this number, 90 were female and 30 were male.

Apparatus. The subject sat $60 \mathrm{~cm}$ in front of a $60 \times 60 \mathrm{~cm}$ square panel covered with black felt cloth and containing a $2.7-\mathrm{cm}$-diam aperture at eye level. A disk of white light was projected onto a translucent glass screen behind the aperture. The light source was a light-discrimination apparatus, manufactured by the Lafayette Instrument Company (Model 14011), with a 60-W Sylvania clear Decor Lite (60CA9C/BL). Nine different light intensities, . $188 \mathrm{log}$ units apart were used; they were, from lowest to highest stimulus value (SV1-SV9): 1.97, $3.04,4.69,7.23,11.2,17.2,26.5,40.9$ and $63.0 \mathrm{fL}$. The experiment was conducted in a small, dimly illuminated room. The light reflected from the disk when it was not illuminated was approximately $.01 \mathrm{fL}$.

Procedure. The following instructions were read to the seated subject: "This is an experiment in brightness perception. A light will be presented repeatedly through a small hole in the screen in front of you. Each time it will be presented for $3 \mathrm{sec}$ and may have a different brightness. The first brightness is called the test brightness. Try to remember this brightness because you will have to distinguish it from all the other brightnesses. When you do recognize the test brightness, say 'same.' If a subsequent brightness is different from the test brightness, say 'different.' Remember, each time the light is presented it will stay on for only $3 \mathrm{sec}$ so try to respond while the light is still on. I will tell you whether you are correct on the first few trials; then you will continue without further help. The first light is the test brightness. Keep its brightness in mind. For every light after that, say only 'same' or 'different.' Any questions?" Following any needed clarification of instructions, each subject was shown the appropriate $\mathrm{S}+$ stimulus. Discrimination training, consisting of 24 stimulus presentations, was then carried out. For half the subjects, S+ was SV2 and S- was SV8; these values were reversed for the other half.

There were three major groups $(n=40)$ corresponding to overrepresentation of S+ or of S- (18 of 24 trials) or equal representation of both (12 trials each). Within each group, there were approximateiy three times as many female subjects as males.

During discrimination training, the experimenter provided feedback by saying "correct," "no, that was different from the original," or "no, that was the same as the original," depending on the response. Generalization testing was initiated with no interruption in the procedure: There were six series of all nine test stimuli, and feedback was no longer given. The stimuli were randomized within each series, and several different random sequences of stimuli were employed in each of the groups. In both generalization testing and discrimination training, the stimulus duration was set at $3 \mathrm{sec}$ and the interstimulus interval ranged unsystematically from $2-4 \mathrm{sec}$.

\section{Results}

Discrimination performance did not differ among the three overrepresentation conditions, nor did it depend on whether the $\mathbf{S}+$ was more or less intense than the $\mathbf{S}-$. Analysis of variance of mean number of errors yielded $\mathrm{F}<1$ for both factors. Three subjects who made more than three errors during the 24 discrimination training trials were dropped from the study and replaced.
A gradient of stimulus generalization was constructed for each subject by summing the number of "same" responses over presentations of each of the test stimuli. Gradients were averaged over individuals within each group. In addition, because gradients for groups trained with the more intense stimulus as $\mathbf{S}+$ did not generally differ from those for groups trained with the less intense stimulus as $\mathbf{S}+$, data for the different groups were collapsed over this variable. The group mean gradients for the different overrepresentation groups are presented in Figure 1. Inspection of the figure suggests several conclusions. In comparison with the gradient of the equal representation group, it appears that overrepresentation of $S-$ led to less responding to intermediate stimuli and relatively enhanced responding to stimuli displaced from $\mathrm{S}+$ in the direction opposite from S-. On the other hand, overrepresentation of the $S+$ led to more responding to intermediate stimuli and relatively depressed responding to stimuli displaced from S+ in the direction opposite from S-.

For statistical purposes, the measure used to reflect the shift in the decision criterion was the amount of responding to intermediate stimuli, that is, the mean of responses to SVs $3,4,5,6$, and 7 . Analysis of variance indicated that the differences among the three groups on this measure were highly significant $[F(2,117)=10.58, p<.01]$. Because the direction of group differences was predicted a priori, a one-tailed test of their significance is appropriate. Such tests revealed that the mean of the equal representation group was significantly different from that of both the $S$ - overrepresentation group $[t(117)=1.76$,

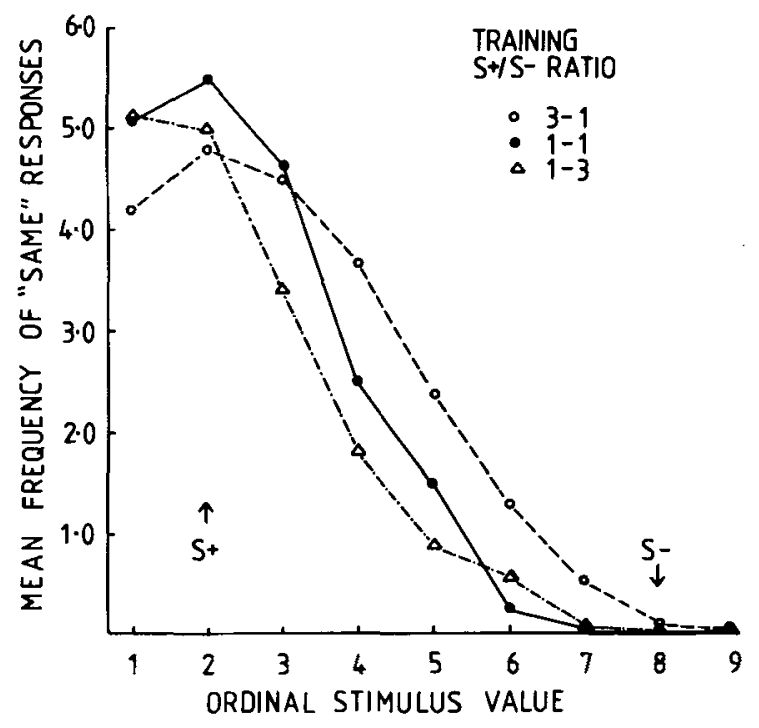

Figure 1. Mean frequency of "same" responses as a function of test stimulus value in generalization testing following training with different ratios of $S+/ S-$ presentation frequency. In training, $S+$ was SV2 and S- was SV8 for half of the subjects; the reverse was true for the other half. 
$\mathrm{p}<.05]$ and the $\mathrm{S}+$ overrepresentation group $[\mathrm{t}(117)=$ $5.53, \mathrm{p}<.01]$.

\section{Discussion}

Experiment 1 tested the predictions that overrepresenting $\mathbf{S}+$ in training should produce a shift in the gradient away from $S-$, whereas overrepresenting $S-$ should produce a shift towards $S-$. Both predictions were confirmed. Thus, Experiment 1 replicated and extended the results of the Thomas and Vogt (1983) experiment. The enhanced responding to values intermediate between $\mathrm{S}+$ and $\mathbf{S}-$ produced by overrepresenting $\mathbf{S}$ - in training, as found in both experiments, seems clearly contradictory to a gradient interaction interpretation. An increase in inhibition should reduce, rather than enhance, responding to intermediate stimuli.

Although the procedure used in the Thomas and Vogt (1983) study and in Experiment 1 was suggested by TSD, the results can also be accounted for by AL theory. That is, overrepresentation of $\mathrm{S}-$ in training should establish a training AL closer to the $S$ - value and overrepresentation of $S+$ should place AL closer to the $S+$ value relative to an equal representation condition. In testing, the AL of each overrepresentation group will therefore shift toward the value that was not overrepresented in training. If we assume that subjects will, in testing, continue to respond maximally to the stimulus that bears the same relationship to $\mathrm{AL}$ that $\mathrm{S}+$ did during training, then the $S-$ overrepresentation group should show reduced responding to intermediate values and a shift in the generalization gradient away from $S-$, whereas the $S+$ overrepresentation group should show increased responding to intermediate stimuli and a shift in the gradient towards $S-$. The gradient for a group in which $S+$ and $S-$ are presented equally often in training should fall midway between gradients for the $\mathbf{S}+$ and $\mathbf{S}$ - overrepresentation groups. This is, of course, what was observed in the present experiment.

Because the AL interpretation of the present results is based on postulated changes in AL that occur during the course of testing, it would be useful to show that the gradients of the three groups are comparable at the beginning of generalization testing and diverge as testing progresses. Unfortunately, however, the shift in $\mathrm{AL}$ and in the location of the gradient often occurs within the first few stimulus presentations, so a comparison of gradients based on the entire first series and those based upon subsequent series may fail to show systematic differences. In order to compute entire gradients based on the first few stimulus presentations given to each subject, the data must be combined from a large number of subjects. In studies by Thomas and Fenner (1978) and by Thomas, Strub, and Dickson (1974), group sizes were sufficiently large (140 and 160 , respectively) for trial-by-trial gradient shifts during testing to be demonstrated. Newlin, Rodgers, Dickson, Strub, and Thomas (1978) found that measured changes in AL during testing, across different experimen- tal groups, were correlated with observed differences in the locus of maximal generalized responding in those groups. With 40 subjects per group in the present experiment, however, a trial-by-trial analysis of generalized responding was not possible.

The results of the Thomas and Vogt (1983) study and of the present Experiment 1 cannot distinguish between TSD and AL positions. An alternative design is required in which the two positions make clearly differing predictions. Thus, Experiment 2 varied the frequency with which $\mathrm{S}+$ and $\mathrm{S}-$ were presented during testing rather than during training. For all groups, $\mathbf{S}+$ and $\mathbf{S}-$ appeared equally often in training (i.e., on 12 trials each). For one group, S+ was overrepresented on test trials; for a second group, $\mathbf{S}-$ was overrepresented; for the third group, each test stimulus appeared equally often. According to AL theory, overrepresenting the S+ during testing should result in a shift in AL toward the $S+$ value, with a resulting decrease in responding to intermediate stimuli and a shift in the gradient away from S-. Note that these effects are opposite to those predicted for overrepresenting the S+ in training in the Thomas and Vogt (1983) study and in Experiment 1. The logic of the prediction is straightforward: AL moves toward the overrepresented value. If overrepresentation occurs during testing, the $A L$ shift is reflected directly in test responding. If overrepresentation occurs during training, then during the test, without overrepresentation, AL will shift away from the previously overrepresented value.

The theory of signal detection predicts that overrepresentation of a value during testing will have the same effect that it does during training, because the only factor influencing location of response criteria is relative presentation frequency of test stimuli during the test (Green \& Swets, 1974). According to TSD, overrepresenting S+ during testing (as during training) should shift the decision criterion toward the $S-$ value, thereby increasing the tendency to respond to intermediate values and shifting the gradient towards $\mathrm{S}-$. Thus, the two theories make opposite predictions when overrepresentation occurs during testing, whereas they make the same predictions when it occurs during training.

\section{EXPERIMENT 2}

\section{Method}

Subjects. The subjects were 120 students enrolled in introductory psychology classes at the University of Colorado. Of this number, 90 were female and 30 were male.

Apparatus. The apparatus was the same as in Experiment 1.

Procedure. The procedure was the same as that used in Experiment 1 with the exception that the three major groups differed in frequency of presentation of the training stimuli during the generalization test rather than during discrimination training. For one of the groups, the $S+$ value appeared on $56 \%$ of all test trials (i.e., 60 of 108), whereas each other stimulus appeared on 5\% (i.e., 6 times). There were six blocks of test stimuli arranged in a nonsystematic order, except that the overrepresented value occurred 10 times, whereas each other value occurred once. For a second 
group, the $S-$ value occurred 60 times during the test. For a third group, each of the six test blocks included one presentation of each of the nine test stimuli. That is, test stimuli were equally represented. For half of the subjects, the more intense value was $\mathbf{S +}$; for half, it was S-. Each of the six groups contained approximately three times as many female subjects as males.

\section{Results}

Three subjects who made more than three errors during the 24 discrimination training trials were dropped from the study and replaced. Discrimination performance (i.e., number of errors) did not depend on whether the $S+$ was more or less intense than the $S-(t<1)$.

A gradient of stimulus generalization was constructed for each subject by calculating the number of "same" responses made to each of the test stimuli, corrected for differences in opportunity to respond in the case of overrepresented stimuli. This correction was simply to divide the number of responses to the overrepresented stimulus by 10 . Because the gradients of subjects for which $S+$ was SV2 were comparable to those for which $S+$ was SV8, the subgroups were pooled for the figure and for statistical analysis. The mean gradients of the different overrepresentation groups are presented in Figure 2.

Inspection of Figure 2 reveals that overrepresenting Sin testing strongly enhanced responding to intermediate stimulus values and shifted the gradients in the direction of $S-$. Indeed, it produced a reverse peak shift (toward $\mathrm{S}-$ ) in both cases (i.e., with both the subgroup for which SV2 was $S+$ and that for which SV8 was S+). The equal representation gradient fell between those of groups in

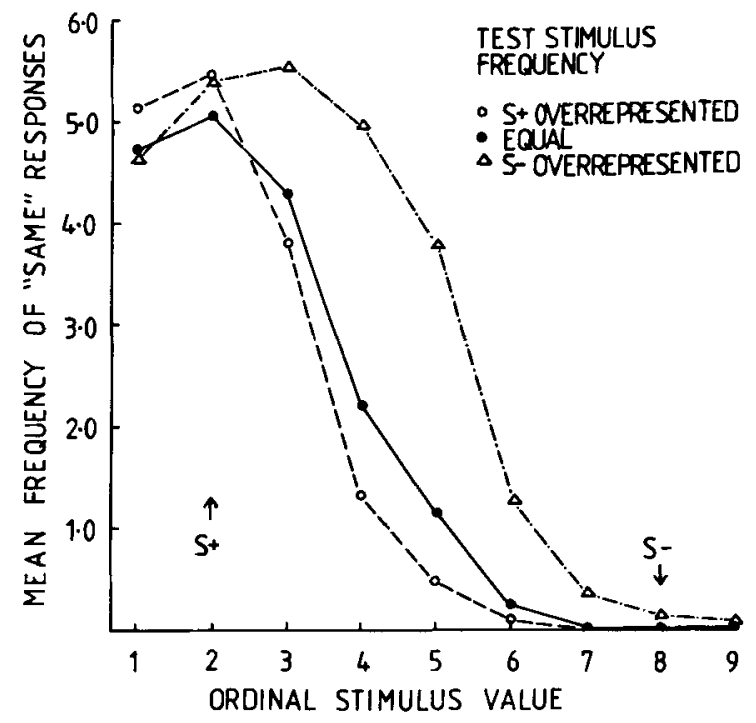

Figure 2. Mean frequency of "same" responses as a function of test stimulus value in generalization testing, in which $S+$ and $S-$ stimuli were equally or overrepresented. Response frequencies for overrepresented stimuli were corrected for stimulus presentation frequency. In training, S+ was SV2 and S- was SV8 for half of the subjects; the reverse was true for the other half. which $\mathbf{S}+$ or $\mathbf{S}-$ were overrepresented. When $\mathbf{S}+$ was overrepresented in the test, the gradient also tended to sharpen compared to the gradient for the equalrepresentation condition.

The data of Experiment 2 were analyzed in the same manner as in Experiment 1 except that two-tailed tests were used for individual comparisons. Analysis of variance of mean number of responses to intermediate stimulus values showed the main effect of overrepresentation to be highly significant $[F(2,117)=71.37, p<.01]$. Subsequent tests indicated that the mean for the equal representation group was significantly different from that of the $S-$ overrepresentation group $[t(117)=2.45$, $p<$ $.01]$ and that of the $S+$ overrepresentation group $[t(117)$ $=8.90, \mathrm{p}<.01]$.

\section{Discussion}

The major findings of Experiment 2 are consistent with predictions based on AL theory but not those based on TSD. Overrepresenting $S-$ in testing resulted in more, rather than less, responding to intermediate stimuli and a shift in the gradients towards S-. Gradients for the equal representation condition generally fell between the other two, indicating that both overrepresenting $S+$ and overrepresenting $S-$ during testing were effective manipulations. In addition, overrepresentation of $S+$ sharpened the gradients. The source of this additional effect is unclear at the present time and requires further examination under conditions in which degree of overrepresentation is varied. The direction of shifts with overrepresentation does, however, lend unequivocal support to the AL interpretation.

\section{GENERAL DISCUSSION}

Our results have some interesting implications for the study of signal presentation probability effects on performance in signal detection procedures in that the influence of biasing variables may depend on the incorporation of feedback in the procedure. This is suggested by the difference in the effects of signal presentation probability in Experiment 1, when it was manipulated during training when feedback was available, and Experiment 2, when it was manipulated during testing without feedback. Although signal presentation probability has been assumed to be an important determinant of bias (Dusoir, 1975, 1983; Green \& Swets, 1974), the nature of its interaction with feedback or payoff contingencies is unclear. Payoff also has a strong influence on bias (McCarthy \& Davison, 1981), and the present data suggest that this influence may be mediated by signal presentation probability.

The fact that overrepresentation of a stimulus has opposite effects on generalization depending on whether it occurs during training, when feedback is given, or during testing, when no feedback is given, has some parallels in the signal detection literature. The effect of feedback often depends on the way in which it is combined with 
other variables. For example, several studies have reported that (correct) feedback facilitates detection performance when the discrimination is a difficult one, but actually impedes performance on an easy discrimination (Carterette, Friedman, \& Wyman, 1966; Carterette \& Wyman, 1962; Gundy, 1961).

The main contribution of TSD to this research was to suggest an important role for relative frequency of presentation of particular training or test stimuli. These variables did have consistent and significant effects, although not always in the direction predicted by the theory. In general, AL did a better job of predicting our subjects' behavior than did TSD, although neither formulation can provide a complete account of the extant data. Perhaps we have erred in oversimplifying what our subjects learn in these experimental situations. The AL theory views all judgments as relative, implying that the subject has no knowledge of the absolute values of the training stimuli, whereas TSD presumes no knowledge of their relative values. A parallel exists in the province of animal discrimination learning, where Spence (1937) claimed that learning was always absolute and Krechevsky (1938) proposed that it was always relative. Premack (1978) has recently argued that animals may learn both the absolute and relative attributes of the training stimuli, with the emphasis on one or the other depending upon the species, the test conditions, the stimulus dimension, and a host of other factors. Newlin et al. (1979) recognized this and proposed, for example, that subjects initially may encode the stimuli relationally and then learn about absolute attributes with more extended training. Perhaps a useful strategy will be to follow their lead and to seek to discover the conditions that determine whether absolute or relational encoding of stimuli predominates in the judgments subjects make in the human voluntary generalization situation.

\section{REFERENCES}

Baron, A. (1973). Postdiscrimination gradients on a tone continuum. Journal of Experimental Psychology, 101, 337-342.

BLougH, D. S. (1969). Generalization gradient shape and summation in steady-state test. Journal of the Experimental Analysis of Behavior, 12, 91-104.

Boneau, C. A., Cole, J. L. (1967). Decision theory, the pigeon, and the psychophysical function. Psychological Review, 74, 123-135.

Brown, J. S., Bilodeau, E. A., \& Baron, M. R. (1951). Bidirectional gradients in the strength of a generalized voluntary response to stimuli on a visual-spatial dimension. Journal of Experimental Psychology, 41, 52-61.

Carterette, E. C., Friedman, M. P., Wyman, M. J. (1966). Feedback and psychophysical variables in signal detection. Journal of the Acoustical Society of America, 39, 1051-1055.

Carterette, E. C., Wyman, M. J. (1962). Applications of a Markov learning model to a simple detection situation involving social pressure. In J. H. Criswell, H. Solomon, \& P. Suppes (Eds.), Mathe- matical methods in small group processes. Stanford, CA: Stanford University Press.

DoLL, T. J., \& ThомAs, D. R. (1967). Effects of discrimination training on stimulus generalization for human subjects. Journal of Experimental Psychology, 75, 508-512.

Dusolr, T. (1975). Treatment of bias in detection and recognition models: A review. Perception \& Psychophysics, 17, 167-178.

Dusolr, T. (1983). Isobias curves in some detection tasks. Perception \& Psychophysics, 33, 403-412.

GALZIO, M., \& BARON, A. (1979). Human postdiscrimination gradients: The effects of three-stimulus discrimination training. Animal Learning \& Behavior, 7, 53-56.

GREEN, D. M., \& SwETS, J. A. (1974). Signal detection theory and psychophysics. Huntingdon, NY: Krieger.

GUNDY, R. F. (1961). Auditory detection of an unspecified signal. Journal of the Acoustical Society of America, 33, 1008-1012.

HELSON, H. (1964). Adaptation level theory. New York: Harper \& Row.

KALISH, H. I. (1958). The relationship between discriminability and stimulus generalization: A re-evaluation. Journal of Experimental Psychology, 55, 637-644.

KRECHEVSKY, I. (1938). A study of the continuity of the problem solving process. Psychological Review, 45, 107-133.

MCCARTHY, D., \& DAvison, M. (1981). Towards a behavioural theory of bias in signal detection. Perception \& Psychophysics, 29, 371-382.

Newlin, R. J., Rodgers, J. P., Dickson, J. R., Strub, H., \& Thomas, D. R. (1978). The central tendency effect in stimulus generalization: Effects of establishing a "preexperimental" frame of reference. Perception \& Psychophysics, 24, 161-167.

Newlin, R. J., Rodgers, J. P., \& Thomas, D. R. (1979). Two determinants of the peak shift in human voluntary stimulus generalization. Perception \& Psychophysics, 25, 478-486.

Premack, D. (1978). On the abstractness of human concepts: Why it would be difficult to talk to a pigeon. In Hulse, S. H., Fowler, H., \& Honig, W. K. (Eds.), Cognitive processes in animal behavior. Hillsdale, NJ: Erlbaum.

Purtle, R. B. (1973). Peak shift: A review. Psychological Bulletin, 80, 408-421.

Schulman, A. I., \& Greenberg, G. Z. (1970). Operating characteristics and the prior probability of the signal. Perception \& Psychophysics, 8, 317-320.

SPENCE, K. W. (1937). The differential response in animals to stimuli varying within a single dimension. Psychological Review, 44, 430-444.

Thomas, D. R. (1974). The role of adaptation-level in stimulus generalization. In G. H. Bower (Ed.), The psychology of learning and motivation (Vol. 8). New York: Academic Press.

Thomas, D. R., \& FENNER, D. P. (1978). The central tendency effect in stimulus generalization: The effect of sex of subject. American Journal of Psychology, 91, 509-522.

Thomas, D. R., Strub, H. , Dickson, J. F., JR. (1974). Adaptation level and the central tendency effect in stimulus generalization. Journal of Experimental Psychology, 103, 466-474.

Thomas, D. R., Svinicki, M. D., \&ogt, J. (1973). Adaptation-level as a factor in human discrimination learning and stimulus generalization. Journal of Experimental Psychology, 97, 210-219.

THOMAs, D. R., \& VoGT, M. B. (1983). Stimulus generalization in humans viewed from a signal detection perspective. New Zealand Journal of Psychology, 12, 1-6.

White, K. G., \& Thомаs, D. R. (1979). Postdiscrimination generalization and adaptation level in humans: An extension of Galizio and Baron. Animal Learning \& Behavior, 7, 564-565.

(Manuscript received February 2, 1984; revision accepted for publication February 14, 1985.) 